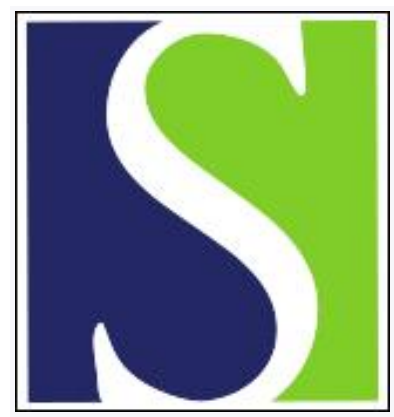

Scand J Work Environ Health 2016;42(4):291-298

https://doi.org/10.5271/sjweh.3563

Published online: 21 Apr 2016, Issue date: 01 Jul 2016

Self-reported occupational physical activity and cardiorespiratory fitness: Importance for cardiovascular disease and all-cause mortality

by Holtermann A, Marott JL, Gyntelberg F, Søgaard K, Mortensen OS, Prescott E, Schnohr P

Workers with high occupational physical activity and low cardiorespiratory fitness were found to have a strong excessive risk for CVD mortality. This supports the classic, but scarcely documented thesis in occupational medicine, of the importance of cardiorespiratory fitness for tolerating high physical work demands. Preventive measures for reducing excessive cardiovascular work strain and CVD risk are needed.

Affiliation: National Research Centre for the Working Environment, Lerso Parkallé 105, DK-2100 Copenhagen, Denmark. aho@nrcwe.dk

Refers to the following texts of the Journal: 2007;33(6):405-424

2009;35(6):466-474 1984;10(6):403-408 2010;36(5):357-365

2010;36(5):349-355 2012;38(5):467-475 2015;41(2):124-139

The following articles refer to this text: 2018;44(2):202-211; 2022;48(2):86-98; 2023;49(3):231-244

Key terms: all-cause mortality; cardiorespiratory fitness; cardiovascular disease; CVD; heart disease; mortality; occupational physical activity; physical activity; physical fitness; physical work demand; self-report

This article in PubMed: www.ncbi.nlm.nih.gov/pubmed/27100403

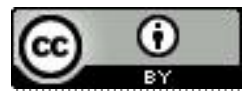




\title{
Self-reported occupational physical activity and cardiorespiratory fitness: Importance for cardiovascular disease and all-cause mortality
}

\author{
by Andreas Holtermann, PhD, ${ }^{1,2}$ Jacob Louis Marott, MSc, ${ }^{3}$ Finn Gyntelberg, DrMed, ${ }^{3,4}$ Karen Søgaard, \\ PhD, ${ }^{2}$ Ole Steen Mortensen, PhD, ${ }^{5}$ Eva Prescott, DrMed, ${ }^{3,6}$ Peter Schnohr, DrMed ${ }^{3}$
}

\begin{abstract}
Holtermann A, Marott JL, Gyntelberg F, Søgaard K, Mortensen OS, Prescott E, Schnohr P. Self-reported occupational physical activity and cardiorespiratory fitness: Importance for cardiovascular disease and all-cause mortality. Scand J Work Environ Health. 2016;42(4):291-298. doi:10.5271/sjweh.3563
\end{abstract}

\begin{abstract}
Objectives This study aimed to investigate whether workers with the combination of high occupational physical activity (OPA) and low cardiorespiratory fitness have an increased risk of cardiovascular disease (CVD) and all-cause mortality.

Methods Using multivariable Cox proportional hazards regression, we analyzed 2190 males and 2534 females from the Copenhagen City Heart Study, aged 20-67 years and with no known CVD at study entry in 1991-1994, for the risk of CVD and all-cause mortality from independent, stratified and combinations of self-reported OPA (ie, low, moderate and high) and cardiorespiratory fitness (low, same and higher as peers) at baseline.

Results During a median follow-up of 18.5 years, 257 and 852 individuals died from CVD and any cause, respectively. In the fully-adjusted model, an increased risk for CVD mortality was found for those with low compared to high self-reported cardiorespiratory fitness [hazard ratio (HR) 2.17, $95 \%$ confidence interval $(95 \%$ CI 1.40-3.38], for those with high compared to low OPA (HR 1.45, 95\% CI 1.05-2.00), and for those with high compared to low OPA within the strata of low self-reported cardiorespiratory fitness (HR 2.83, 95\% CI 1.24-6.46). Moreover, those with the combination of high OPA and low self-reported cardiorespiratory fitness had an increased risk for CVD mortality compared to those with the combination of low OPA and high selfreported cardiorespiratory fitness (HR 6.22, 95\% CI 2.67-14.49). Rather similar, but lower risk estimates were found for all-cause mortality.
\end{abstract}

Conclusion These findings may have important implications for CVD prevention among workers with excessive cardiovascular strain at work.

Key terms CVD; physical work demand; heart disease; physical fitness.

A classic notion in occupational medicine is that the combination of high occupational physical activity (OPA) and low cardiorespiratory fitness imposes an excessive physiological strain (1), which may lead to endothelium dysfunction, atherosclerosis and consequently cardiovascular disease (CVD) (2). This is illustrated by the higher relative cardiovascular load for a given work task experienced by a worker with low compared to high cardiorespiratory fitness (3). Interna- tional guidelines favor a balanced relationship between the OPA and cardiorespiratory fitness in order to reduce the overall risk of cardiovascular overstrain of workers $(4,5)$. However, only very few previous prospective studies have investigated the international guidelines in relation to CVD or all-cause mortality $(3,6,7)$. Since a considerable fraction of the general population still has a physically demanding job involving much walking, standing and lifting (8), further investigations on the

1 The National Research Centre for the Working Environment, Copenhagen, Denmark.

2 Institute of Sports Science and Clinical Biomechanics, University of Southern Denmark, Odense M, Denmark.

3 The Copenhagen City Heart Study, Frederiksberg Hospital, Copenhagen, Denmark.

4 The Copenhagen Male Study, Epidemiological Research Unit, Department of Occupational and Environmental Medicine, Bispebjerg University Hospital, Copenhagen NV, Denmark.

5 Department of Occupational and Social Medicine, Copenhagen University Hospital Holbæk, 4300 Holbæk. Denmark.

6 Department of Cardiology, Bispebjerg University Hospital, Copenhagen, Denmark.

Correspondence to: Andreas Holtermann, Professor, PhD, National Research Centre for the Working Environment, Lerso Parkallé 105, DK2100 Copenhagen, Denmark. [E-mail: aho@nrcwe.dk] 
significance of the balance between OPA and cardiorespiratory fitness on the risk of CVD are needed.

Self-reported cardiorespiratory fitness has been shown to have a generally good validity and reliability compared with physiological tests $(9,10)$, be a strong independent predictor for CVD mortality (11) and thus be a good alternative when cardiorespiratory fitness cannot be physiologically measured in large surveys (9). Therefore, the use of self-reported data allows for an investigation of the relevant analysis of the combined effects of OPA and self-reported cardiorespiratory fitness on cardiovascular and all-cause mortality in a prospective population study.

\section{Methods}

\section{Study design and population}

The Copenhagen City Heart Study is a prospective population study in which a random sample of the population living in an area of Copenhagen is invited to participate at regular intervals. Details of the enrolment and examinations are described elsewhere (12).

Between 1991-1994, 10135 participants completed a self-administrated questionnaire and participated in a physical examination including the same standardized and validated methods as previously described in detail (12). Participants with previous myocardial infarction or stroke, either by self-report or ascertained through the Danish National Patient Register from establishment of the register in 1977 until participant study inclusion, were excluded $(\mathrm{N}=793)$. Participants were excluded if older than the retirement age of 67 years $(\mathrm{N}=3087)$, or missing information on OPA $(\mathrm{N}=1500)$ or self-reported cardiorespiratory fitness $(\mathrm{N}=31)$, leaving $(\mathrm{N}=4724)$ persons eligible for analyses.

\section{Ethical approval}

The Committee of Biomedical Research Ethics for the Capital region in Denmark approved the study (H-KF01-144/01). All data was de-identified and analyzed anonymously. The participants provided written consent to participate in the study. The ethics committee approved this consent procedure.

\section{Predictive variables}

A single question was applied for measuring OPA: "Which description most precisely covers your pattern of physical activity at work?" (13). The four possible answers included: (i) "You are mainly sedentary and do not walk much around at your workplace, eg, desk work, work including assembling of minor parts" [score
1]; (ii) "You walk around quite a bit at your workplace but do not have to carry heavy items, eg, light industrial work, non-sedentary office work, inspection and the like" [score 2]; (iii) "Most of the time you walk, and you often have to walk upstairs and lift various items. Examples include mail delivery and construction work" [score 3]; and (iv) "You have heavy physical work. You carry heavy burdens and carry out physically strenuous work, eg, work including digging and shoveling" [score 4]. Due to the low number of participants in the highest category of OPA, the variable was categorized into: score 1=low, score 2=moderate, and score 3-4=high.

To measure self-reported cardiorespiratory fitness, a single question was use: "How do you rate your cardiorespiratory fitness compared to your peers?". The response categories were: "same", "higher" or "lower" (11) and referred to as "moderate", "high" and "low" in the manuscript.

In the combined variable of OPA and cardiorespiratory fitness, all potential combinations between the low, moderate and high OPA, and low, moderate and high cardiorespiratory fitness generated the nine categories.

\section{Covariates}

Information on smoking habits was based on a singleitem question, and the study participants were categorized as never smokers, ex-smokers, and current smokers of $1-14$, or $\geq 15$ cigarettes per day. Information on alcohol consumption was self-reported, and the study participants were categorized in the statistical analyses as abstainers, or monthly, weekly, or daily consumers. Household income was based on self-reported average income per month within the last year and categorized as low, medium, and high. Diabetes was self-reported or a non-fasting blood glucose $\geq 11$.1. Treatment for hypertension was self-reported, and categorized as yes/no. Systolic blood pressure was measured in a sitting position after five minutes of rest, and applied as a continuous variable in the statistical analysis. Body mass index (BMI) was calculated and categorized as underweight, normal, overweight and obese $\left(<18.5,18.5-24.9,25-29.9, \geq 30 \mathrm{~kg} / \mathrm{m}^{2}\right.$, respectively). Cholesterol was measured non-fasting in $\mathrm{mmol} / \mathrm{l}$. A single question regarding leisure-time physical activity with four answer options was applied for measuring leisure time physical activity (13). Self-rated health was measured by the question: "How would you rate your health the past year?" with response categories "very good", "good", "poor" and "very poor".

\section{Follow-up}

Follow-up was carried out by data linkage to national registers. Deaths were obtained until April 2013 from The Civil Registration System and causes of death from 
The National Register of Causes of Death. CVD death was defined as ICD-8: 390-458 and ICD-10: I00-I99. The median duration of follow-up was 18.5 (range 0-20.2) years for CVD death and 19.8 (range 0-21.5) years for all-cause mortality.

\section{Statistical analysis}

The associations between the independent, stratified and combined variable of OPA and cardiorespiratory fitness and the respective outcomes (ie, CVD mortality and all-cause mortality) were studied using Cox proportional hazards regression models. The Cox models were performed with age as underlying time scale and age at baseline as entry time. Two Cox models with forced entry of the following covariates were performed: model A (age and sex) and model B (model A + leisure-time physical activity, BMI, smoking, household income, alcohol consumption and diabetes).

Uni- and multi-adjusted Cox proportional hazards regression analyses were performed with a multiplicative interaction term between OPA, self-reported cardiorespiratory fitness and the outcomes CVD and all-cause mortality, respectively. Moreover, the multiplicative interaction for sex was tested in the same model.
The assumption of proportionality in the Cox regression models was tested with the Lin, Wei, and Ying score process test (14). Misspecifications of the functional form of the covariates were tested by plotting the continuous covariates against the cumulative residuals and comparing them to random realizations under the model. $\mathrm{P}<0.05$ was considered statistically significant. Statistical analyses were performed with $\mathrm{R}$ version 3.0.1.

\section{Results}

During a median follow-up of 18.5 years (range 0-20.2) for CVD mortality and 19.8 years (range $0-21.5$ ) for all-cause mortality, 852 (males 489) died from CVD and 257 (males 158) from all-causes.

Tables 1a and b shows demographic, lifestyle, and clinical factors according to the nine categories of combined levels of OPA and self-reported cardiorespiratory fitness among 2190 males and 2534 females without a history of cardiovascular disorders in 1991-94 in the Copenhagen City Heart Study. Several numerical differences in self-reported cardiorespiratory fitness were observed. Persons with low self-reported

Table 1a. Baseline characteristics of study participants stratified on self-reported cardiorespiratory fitness and occupational physical activity (OPA) among 2190 males and 2534 females without a history of cardiovascular disorders in the Copenhagen City Heart Study in 1991-1994. [SD=standard deviation; BMI=body mass index].

\begin{tabular}{|c|c|c|c|c|c|c|c|c|c|}
\hline & \multicolumn{9}{|c|}{ Self-reported cardiorespiratory fitness } \\
\hline & \multicolumn{3}{|c|}{ High } & \multicolumn{3}{|c|}{ Moderate } & \multicolumn{3}{|c|}{ Low } \\
\hline & \multicolumn{3}{|c|}{ OPA } & \multicolumn{3}{|c|}{ OPA } & \multicolumn{3}{|c|}{ OPA } \\
\hline & $\begin{array}{c}\text { Low } \\
(\mathrm{N}=408)\end{array}$ & $\begin{array}{c}\text { Moderate } \\
(\mathrm{N}=405)\end{array}$ & $\begin{array}{c}\text { High } \\
(\mathrm{N}=433)\end{array}$ & $\begin{array}{c}\text { Low } \\
(\mathrm{N}=956)\end{array}$ & $\begin{array}{c}\text { Moderate } \\
(\mathrm{N}=919)\end{array}$ & $\begin{array}{c}\text { High } \\
(\mathrm{N}=962)\end{array}$ & $\begin{array}{c}\text { Low } \\
(\mathrm{N}=259)\end{array}$ & $\begin{array}{c}\text { Moderate } \\
(\mathrm{N}=215)\end{array}$ & $\begin{array}{c}\text { High } \\
(\mathrm{N}=167)\end{array}$ \\
\hline & $\%$ & $\%$ & $\%$ & $\%$ & $\%$ & $\%$ & $\%$ & $\%$ & $\%$ \\
\hline High leisure-time PA & 67.3 & 59.7 & 66.4 & 32.8 & 29.8 & 34.7 & 12.1 & 15.8 & 24.0 \\
\hline Outstanding self-rated health & 19.7 & 19.6 & 17.0 & 10.2 & 8.0 & 7.0 & 4.3 & 1.9 & 3.6 \\
\hline High household income & 58.7 & 48.0 & 43.8 & 58.2 & 48.7 & 46.1 & 55.2 & 40.0 & 44.1 \\
\hline Obese (BMI $\left.\geq 30 \mathrm{~kg} / \mathrm{m}^{2}\right)$ & 3.7 & 5.7 & 7.6 & 9.6 & 10.8 & 13.8 & 18.1 & 25.4 & 20.4 \\
\hline Never smoking & 36.5 & 32.5 & 26.9 & 31.3 & 28.7 & 21.3 & 22.5 & 26.2 & 19.4 \\
\hline Hypertensive medicine & 2.5 & 3.5 & 3.5 & 5.6 & 5.9 & 5.6 & 9.8 & 9.4 & 5.4 \\
\hline Daily alcohol consumption & 15.0 & 23.3 & 25.2 & 18.7 & 18.0 & 21.9 & 18.1 & 19.2 & 13.2 \\
\hline Diabetes & 1.0 & 0.5 & 1.8 & 2.0 & 2.2 & 3.7 & 2.7 & 1.9 & 2.4 \\
\hline
\end{tabular}

Table 1b. Baseline characteristics continued.

\begin{tabular}{|c|c|c|c|c|c|c|c|c|c|c|c|c|c|c|c|c|c|c|}
\hline & \multicolumn{18}{|c|}{ Self-reported cardiorespiratory fitness } \\
\hline & \multicolumn{6}{|c|}{ High } & \multicolumn{6}{|c|}{ Moderate } & \multicolumn{6}{|c|}{ Low } \\
\hline & \multicolumn{6}{|c|}{ OPA } & \multicolumn{6}{|c|}{ OPA } & \multicolumn{6}{|c|}{$\mathrm{OPA}$} \\
\hline & \multicolumn{2}{|c|}{$\begin{array}{c}\text { Low } \\
(\mathrm{N}=408)\end{array}$} & \multicolumn{2}{|c|}{$\begin{array}{l}\text { Moderate } \\
(\mathrm{N}=405)\end{array}$} & \multicolumn{2}{|c|}{$\begin{array}{c}\text { High } \\
(\mathrm{N}=433)\end{array}$} & \multicolumn{2}{|c|}{$\begin{array}{c}\text { Low } \\
(\mathrm{N}=956)\end{array}$} & \multicolumn{2}{|c|}{$\begin{array}{c}\text { Moderate } \\
(\mathrm{N}=919)\end{array}$} & \multicolumn{2}{|c|}{$\begin{array}{c}\text { High } \\
(\mathrm{N}=962)\end{array}$} & \multicolumn{2}{|c|}{$\begin{array}{c}\text { Low } \\
(N=259)\end{array}$} & \multicolumn{2}{|c|}{$\begin{array}{c}\text { Moderate } \\
(\mathrm{N}=215)\end{array}$} & \multicolumn{2}{|c|}{$\begin{array}{c}\text { High } \\
(N=167)\end{array}$} \\
\hline & Mean & SD & Mean & $\overline{S D}$ & Mean & SD & Mean & $\overline{\mathrm{SD}}$ & Mean & $\overline{\mathrm{SD}}$ & Mean & $\overline{S D}$ & Mean & $\overline{S D}$ & Mean & SD & Mean & SD \\
\hline Age (years) & 46.2 & 12.1 & 49.3 & 11.5 & 48.2 & 11.0 & 45.1 & 11.8 & 48.3 & 11.0 & 46.6 & 10.8 & 44.6 & 11.5 & 45.5 & 11.8 & 43.0 & 10.9 \\
\hline Systolic blood pressure & 127.7 & 17.7 & 130.0 & 18.3 & 129.6 & 18.9 & 128.5 & 18.8 & 130.2 & 18.9 & 130.1 & 19.0 & 130.0 & 19.4 & 129.0 & 20.7 & 130.3 & 20.3 \\
\hline Total cholesterol $(\mathrm{mmol} / \mathrm{l})$ & 5.6 & 1.2 & 5.9 & 1.2 & 5.8 & 1.2 & 5.9 & 1.3 & 5.9 & 1.3 & 5.8 & 1.3 & 5.8 & 1.2 & 5.9 & 1.3 & 5.8 & 1.4 \\
\hline
\end{tabular}


cardiorespiratory fitness were generally: younger, less frequently having high leisure-time physical activity, less frequently having outstanding self-rated health, less frequently having high household income, more frequently obese, less frequently never smokers, and more frequently taking hypertensive medicine and having diabetes compared with persons with high level of self-reported cardiorespiratory fitness. Fewer differences in OPA were found. Persons with high OPA had less frequently outstanding self-rated health and high household income, were more frequently obese, and were less frequently never smokers compared with persons with low OPA.

Figure 1 illustrates the risk estimates and corresponding forest plots for CVD mortality from the independent and combined categories of OPA and self-reported cardiorespiratory fitness in the univariable and the multivariable Cox regression models. As reported in a previous study (11), those having low self-reported cardiorespiratory fitness had an increased risk for CVD mortality in both the univariable [hazard ratio (HR) $3.27,95 \%$ confidence interval (95\% CI) 2.18-4.90] and multivariable (HR 2.17, 95\% CI 1.40-3.38) models compared to those with high self-reported cardiorespiratory fitness. These estimates remained similar also when including OPA in the models. Those having high OPA had an increased risk for CVD mortality in both the univariable (HR 1.61, 95\% CI 1.19-2.19) and multivariable (HR 1.45, 95\% CI 1.05-2.00) models compared to those with low OPA. These estimates remained similar also when including self-reported cardiorespiratory fitness in the models. In the strata of low self-reported cardiorespiratory fitness, those with high compared to low OPA had a significantly increased risk for CVD mortality in the univariable (HR $2.90,95 \%$ CI 1.42-5.94) and the multivariable (HR 2.83, 95\% CI 1.24-6.46) models. In the strata of moderate self-reported cardiorespiratory fitness, those with high compared to low OPA had a borderline significantly increased risk for CVD mortality in the univariable model (HR 1.46, 95\% CI 1.00-2.13), but not in the multivariable model (HR 1.30, 95\% CI 0.87-1.95). In the strata of high self-reported cardiorespiratory fitness, those with high compared to low OPA had a significantly increased risk for CVD mortality in the univariable model (HR 2.37, 95\% CI 1.06-5.31), but not in the multivariable model (HR 1.66, 95\% CI 0.71-3.86). Those with the combination of high OPA and low self-reported cardiorespiratory fitness had an increased risk for CVD mortality in both the univariable (HR 10.31, 95\% CI 4.50-23.60) and the multivariable (HR 6.22, 95\% CI 2.67-14.49) models compared to those reporting the combination of low OPA and high self-reported cardiorespiratory fitness. These
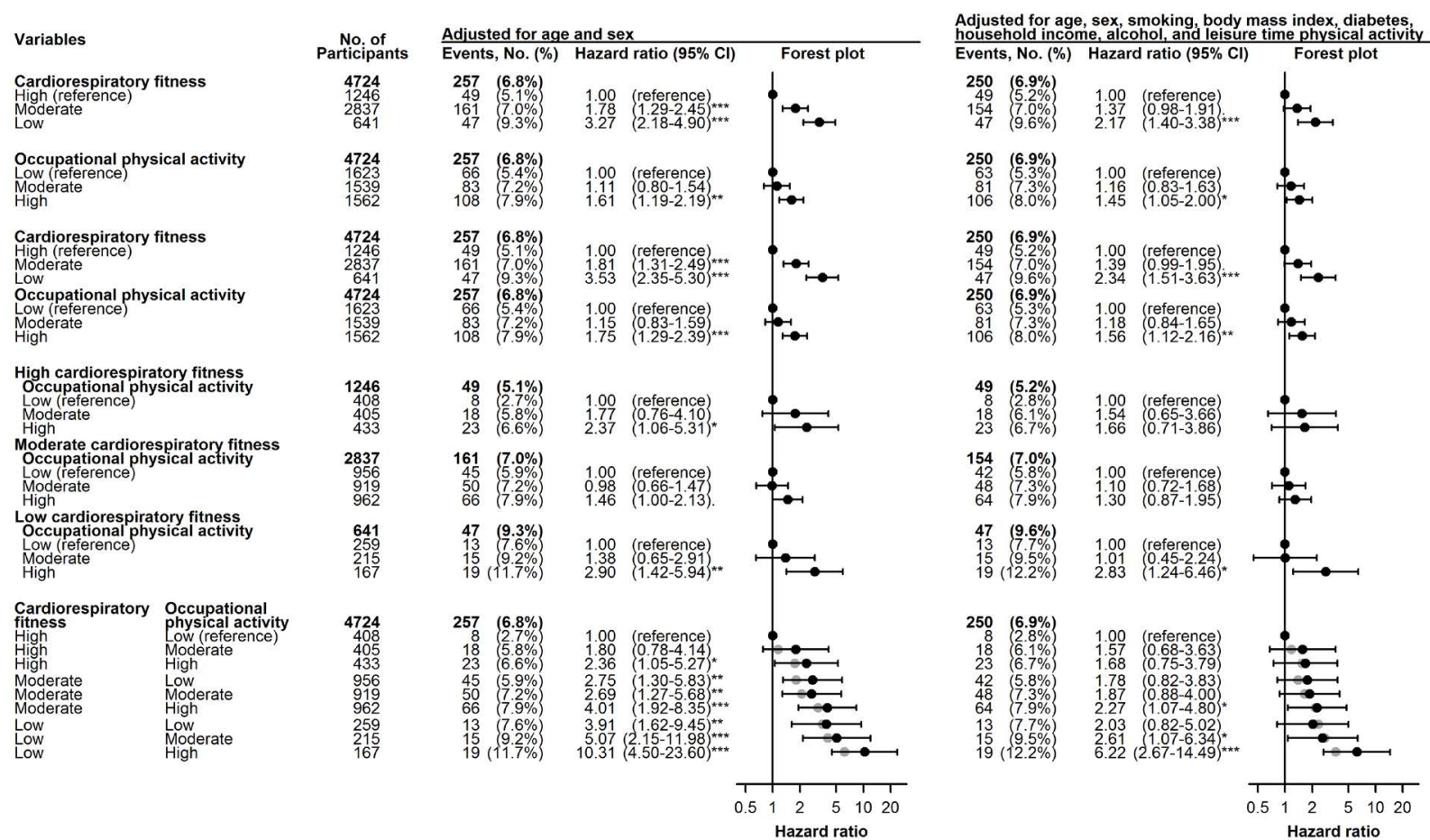

Figure 1. Shows univariable and multivariable Cox proportional hazards regression and Forest plots of the independent, mutually adjusted, stratified and combined categories of self-reported cardiorespiratory fitness and occupational physical activity from 1991-1994 as predictor for cardiovascular disease mortality with median follow-up of 18.5 and 19.8 years among 2190 males and 2534 females without a history of cardiovascular disorders in the Copenhagen City Heart Study. [Percentages are 100\% minus the Kaplan-Meier estimate of being event-free. The grey dots represent the estimated hazard ratios based on the model with the two main effects and no interaction term. $\left.P<0.10 ;{ }^{*} P<0.05 ;{ }^{* *} P<0.01 ;{ }^{* \star *} P<0.001\right]$ 
estimates were mainly similar with the estimates from the model with the two main effects self-reported fitness and OPA and no interaction term between these two (illustrated by grey circles in figure 1).

The risk estimates for the covariates in the full multivariable model for CVD mortality were increased risk for (i) males compared to females (HR 1.97, 95\% CI 1.48-2.62), (ii) obese (BMI $\geq 30 \mathrm{~kg} / \mathrm{m}^{2}$ ) compared to normal BMI (HR 1.94, 95\% CI 1.36-2.77), (iii) current smokers of $\geq 15$ cigarettes/day compared to never smokers (HR 2.75, 95\% CI 1.85-4.07), and (iv) diabetics compared to those without diabetes (HR 2.38, 95\% CI 1.55-3.68). There was no significant increased risk for daily consumers compared to abstainers of alcohol (HR 1.26, 95\% CI 0.81-1.95) and no significant reduced risk for those with high compared to low leisure-time PA (HR 0.74, 95\% CI 0.49-1.13). There was a tendency for a reduced risk for those with high compared to medium household income (HR 0.77, 95\% CI 0.58-1.01).

Figure 2 illustrates the risk estimates and corresponding forest plots for all-cause mortality from the independent and combined categories of OPA and self-reported cardiorespiratory fitness in the univariable and the multivariable Cox regression models. As reported in a previous study (11), those having low self-reported cardiorespiratory fitness had an increased risk for all-cause mortality in both the univariable (HR 2.04, 95\% CI 1.64-2.55) and multivariable (HR 1.57, 95\% CI 1.23-2.00) models compared to those with high self-reported cardiorespiratory fitness. These estimates remained similar also when including OPA in the models. Those having high OPA had an increased risk for all-cause mortality in the univariable (HR 1.23, 95\% CI 1.04-1.45), but not the multivariable model (HR 1.05, 95\% CI 0.88-1.25) compared to those with low OPA. These estimates remained similar also when including self-reported cardiorespiratory fitness in the models. In the strata of low self-reported cardiorespiratory fitness, those with high compared to low OPA did not have an increased risk for all-cause mortality in the univariable (HR 1.06, 95\% CI 0.69-1.62) and multivariable (HR 1.05, 95\% CI 0.65-1.70) models. In the strata of moderate self-reported cardiorespiratory fitness, those with high compared to low OPA had a significantly increased risk for all-cause mortality in the univariable (HR 1.30, 95\% CI 1.06-1.61) but not multivariable (HR $1.07,95 \%$ CI $0.86-1.34$ ) model. In the strata of high self-reported cardiorespiratory fitness, those with high compared to low OPA had a significantly increased risk for CVD mortality in the univariable (HR 1.52, 95\% CI 1.06-2.18) but not multivariable (HR 1.17, 95\% CI 0.79-1.72) model. Those with the combination of high
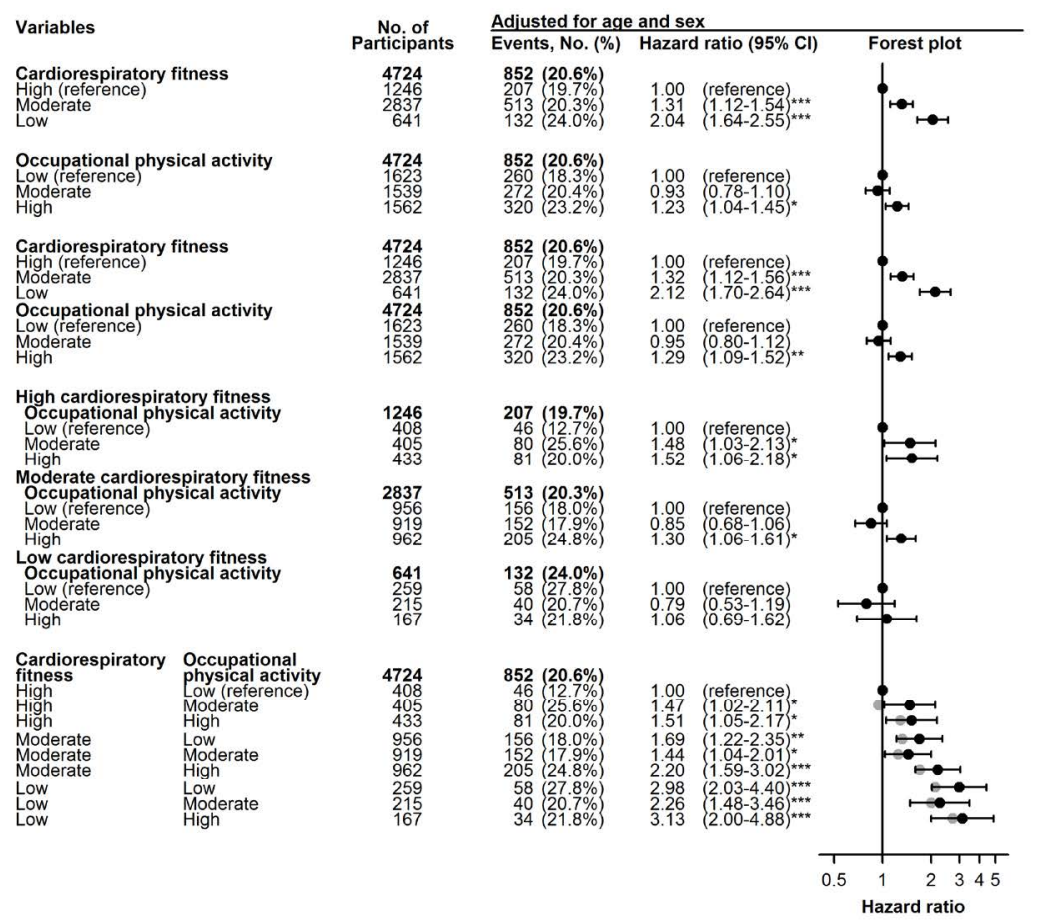

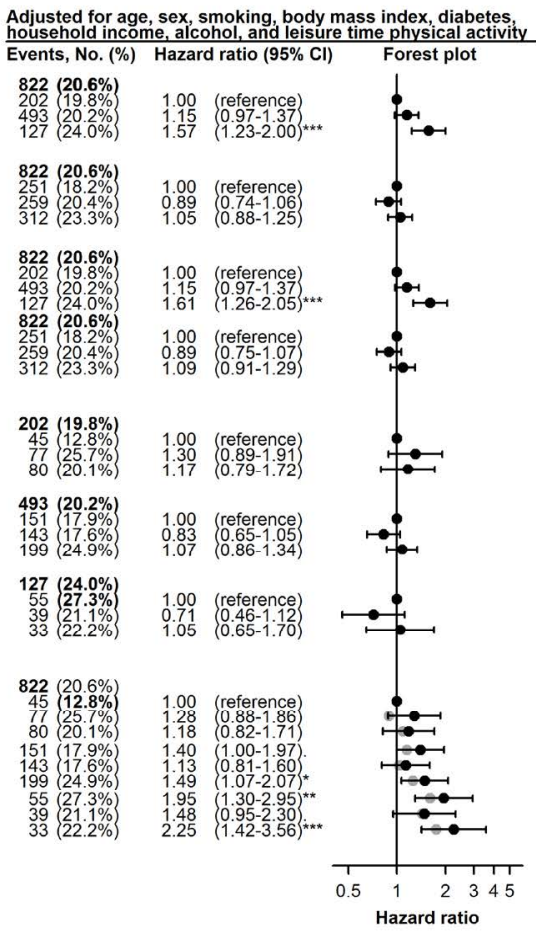

Figure 2. Shows univariable and multivariable Cox proportional hazards regression and Forest plots of the independent, mutually adjusted, stratified and combined categories of self-reported cardiorespiratory fitness and occupational physical activity from 1991-1994 as predictor for all-cause mortality with median follow-up of 18.5 and 19.8 years among 2190 males and 2534 females without a history of cardiovascular disorders in the Copenhagen City Heart Study. [Percentages are 100\% minus the Kaplan-Meier estimate of being event-free. The grey dots represent the estimated hazard ratios based on the model with the two main effects and no interaction term. $P<0.10 ;{ }^{\star} P<0.05 ;{ }^{* *} P<0.01$; ${ }^{* \star}{ }^{*} P<0.001$.] 
OPA and low self-reported cardiorespiratory fitness had an increased risk for all-cause mortality in both the univariable (HR 3.13, 95\% CI 2.00-4.88) and multivariable (HR 2.25, 95\% CI 1.42-3.56) model compared to those reporting the combination of low OPA and high self-reported cardiorespiratory fitness. These estimates were mainly similar with the estimates from the model with the two main effects self-reported fitness and OPA and no interaction term between these two (illustrated by grey circles in figure 2 ).

The risk estimates for the covariates in the full multivariable model for all-cause mortality were increased risk for: (i) males compared to females (HR 1.69, 95\% CI 1.45-1.97), (ii) the obese (BMI $\geq 30 \mathrm{~kg} / \mathrm{m}^{2}$ ) compared to normal BMI (HR 1.36, 95\% CI 1.11-1.68), (iii) current smokers of $\geq 15$ cigarettes/day compared to never smokers (HR 2.89, 95\% CI 2.31-3.61), (iv) daily consumers compared to abstainers of alcohol (tendency for risk) (HR 1.25, 95\% CI 0.99-1.57), and (v) diabetics compared to those without diabetes (HR 1.76, 95\% CI 1.31-2.37). There was no significant reduced risk for those with high compared to low leisure-time PA (HR $0.96,95 \%$ CI $0.76-1.21)$ and a reduced risk for those with high compared to medium household income (HR $0.78,95 \%$ CI $0.66-0.91)$.

Both for CVD mortality and all-cause mortality, no significant interactions between OPA and self-reported cardiorespiratory fitness were found in the multivariable models. Moreover, no significant interactions between OPA and self-reported cardiorespiratory fitness and sex were found in the multivariable model for CVD mortality and all-cause mortality.

\section{Discussion}

The main findings of this study were the increased risk of CVD mortality for those with low compared to high self-reported cardiorespiratory fitness, high compared with low OPA, and high compared with low OPA within the strata of low self-reported cardiorespiratory fitness. Moreover, those with the combination of high OPA and low self-reported cardiorespiratory fitness had an increased risk for CVD mortality compared to those with the combination of low OPA and high self-reported cardiorespiratory fitness. The risk estimates for all-cause mortality were generally similar but somewhat lower than for CVD mortality.

The findings of an increased risk for CVD mortality from high OPA and low cardiorespiratory fitness respectively are in accordance with previous studies on CVD, ischemic disease or mortality $(7,15-18,19)$, and support the importance of these two factors for cardiovascular health. Moreover, in according with previous studies $(3,6,7)$, we found a significant almost three times increased risk for CVD mortality from high OPA among those with low self-reported cardiorespiratory fitness. Among the groups with high and moderate self-reported cardiorespiratory fitness, those with high compared with low OPA had a non-significant increased risk for CVD mortality.

A novel finding of this study was that those with the combination of high OPA and low self-reported cardiorespiratory fitness had more than six times excessive risk (ie, HR 6.22) of CVD mortality and more than doubled risk (ie, HR 2.25) of all-cause mortality than those with the combination of low OPA and high self-reported cardiorespiratory fitness. These excessive risk estimates were found after extensive adjustments for multiple classic risk factors for CVD. Therefore, if these excessive risk estimates hold true, and since a considerable fraction of the population is exposed to high OPA and low self-reported cardiorespiratory fitness, it is likely to contribute significantly to a high incidence of CVD mortality. Also those with the combination of high OPA and moderate self-reported cardiorespiratory fitness were observed to have an increased risk of CVD mortality and all-cause mortality compared to those with the combination of low OPA and high self-reported cardiorespiratory fitness.

The present study corroborates results from other studies on high OPA in relation to low cardiorespiratory fitness also reporting an increased risk on acute myocardial infarction (7), ischemic heart disease (3) and all-cause mortality $(6,3)$ among men free of CVD symptoms at baseline. The physiological underlying mechanisms behind this excessive risk of CVD remain to be documented. A suggested mechanism is that an excessive prolonged cardiovascular strain from high OPA may lead to endothelia dysfunction, which after years of exposure may lead to atherosclerosis (20) and consequently CVD (2). Another potential mechanism is that high OPA leads to elevated blood pressure over the working hours or several blood pressure spikes (eg, due to heavy lifting and/or static postures), which may lead to CVD (21).

\section{Practical implications}

The finding of an increased risk for CVD and all-cause mortality among those with the combination of high OPA and low self-reported cardiorespiratory fitness highlights the importance of the classic notion from work physiology of a balanced relation between OPA and the cardiorespiratory fitness of workers for preserving a good cardiovascular health (22). Our study supports the international guidelines on a balanced relation between OPA and cardiorespiratory fitness for reducing excessive cardiovascular strain of workers and prevent- 
ing CVD $(4,5)$. Restoring a balance between OPA and cardiorespiratory fitness of workers can be attained by reducing the physical workload through ergonomic interventions or organizational initiatives, increasing the cardiorespiratory fitness by physical exercise with sufficient intensity involving dynamic movements of larger muscle groups, and increased recovery (23). There is limited evidence for the effect of ergonomic interventions on reduction of excessive physical workload or improve cardiovascular health (24). Some physical exercise studies have improved cardiorespiratory fitness (25) with consequently decreased cardiovascular strain during work (percentage heart rate reserve) (26). Therefore, we recommend self-reported cardiorespiratory fitness to be included in primary care visits of the general population and occupational health services.

\section{Methodological aspects}

The main strengths of the present study are the large size of a random sample of both men and women representative of the population of Copenhagen in Denmark, the prospective design with a long follow-up time, almost $100 \%$ follow-up on national registers, objective measures of several covariates from clinical examinations, and information on outcomes obtained from valid registers. A limitation of the study is the self-reported exposure measures of OPA and cardiorespiratory fitness, which may entail some degree of misclassification (27). With respect to OPA, such misclassification would, however, not explain the findings presented as the potential bias would rather attenuate the risk estimates. With respect to self-reported cardiorespiratory fitness, such measures have previously been shown to correspond well with physiological tests $(9,10)$ and be a strong independent predictor for CVD mortality (11). Another limitation is the lack of control for psychosocial work factors. However, previous studies have shown that control for psychosocial work factors have minimal influence on the association between OPA and CVD and mortality (20, 8). Moreover, we were not able to differentiate between the OPA questionnaire response categories 3 (ie, mostly including walking activities) and 4 (ie, mostly including heavy physical work with lifting and carrying of heavy burdens) in the statistical analyses due to the low number of participants in the highest category of OPA. This is regarded as an important methodological limitation because much walking may improve cardiovascular health, while static lifting and carrying of heavy objects may increase peak blood pressure transiently to very high levels without improving cardiorespiratory fitness or cardiovascular health.

\section{Concluding remarks}

The main findings of this study were the increased independent risk for CVD mortality among those with low self-reported cardiorespiratory fitness and high OPA. Moreover, an increased risk for CVD mortality was found from high OPA among those self-reporting low cardiorespiratory fitness, and among those with the combination of high OPA and low self-reported cardiorespiratory fitness. These findings may have important implications for occupational medicine and prevention among workers with excessive cardiovascular strain at work. Documented safe and sustainable preventive guidelines and initiatives for reducing excessive cardiovascular work strain and CVD risk among workers are needed.

\section{Acknowledgements}

The Danish Working Environment Foundation funded the study. The authors declare no conflict of interest.

\section{References}

1. Åstrand P-O, Rodahl K. Textbook of work physiology. Physiological bases of exercise. New York: McGraw-Hill Book Company; 1986.

2. Krause N. Physical activity and cardiovascular mortality disentangling the roles of work, fitness, and leisure. Scand J Work Environ Health. 2010;36(5):349-55. http://dx.doi. org/10.5271/sjweh.3077.

3. Holtermann A, Mortensen OS, Burr H, Søgaard K, Gyntelberg F, Suadicani P. Physical demands at work, physical fitness, and 30-year ischaemic heart disease and all-cause mortality in The Copenhagen Male Study. Scand J Work Environ Health. 2010;36(5):357-65. http://dx.doi.org/10.5271/sjweh.2913.

4. Ilmarinen J. Physical load on the cardiovascular-system in different work tasks. Scand J Work Environ Health. 1984;10:403-8. http://dx.doi.org/10.5271/sjweh.2303.

5. Jorgensen K. Permissible Loads Based on Energy-Expenditure Measurements. Ergonomics. 1985;28(1):365-9. http://dx.doi. org/10.1080/00140138508963145.

6. Clays E, Lidegaard M, De Bacquer D, Van Herck K, De Backer G, Kittel F, et al. The combined relationship of occupational and leisure-time physical activity with all-cause mortality among men, accounting for physical fitness. Am J Epidemiol. 2015;179(559):566.

7. Krause N, Brand RJ, Arah OA, Kauhanen J. Occupational physical activity and 20-year incidence of acute myocardial infarction: results from the Kuopio Ischemic Heart Disease Risk Factor Study. Scand J Work Environ Health. 2015;41(2):124-39. http://dx.doi.org/10.5271/sjweh.3476.

8. Holtermann A, Burr H, Hansen JV, Krause N, Søgaard K, 
Mortensen OS. Occupational physical activity and mortality among Danish workers. Int Arch Occup Environ Health. 2012;85(3):305-10. http://dx.doi.org/10.1007/s00420-0110668-x.

9. Keith NR, Clark DO, Stump TE, Miller DK, Callahan CM. Validity and reliability of the Self-Reported Physical Fitness (SRFit) survey. J Phys Act and Health. 2014;11(4):853-9. http://dx.doi.org/10.1123/jpah.2012-0264.

10. Ortega FB, Sanchez-Lopez M, Solera-Martinez M, FernandezSanchez A, Sjöström M, Martinez-Vizcaino V. Self-reported and measured cardiorespiratory fitness similarly predict cardiovascular disease risk in young adults. Scand J Med Sci in Sports 2013;23(6):749-757. http://dx.doi.org/10.1111/j.16000838.2012.01454.x.

11. Holtermann A, Marott JL, Gyntelbe F, Søgaard K, Mortensen OS, Prescott E, et al. Self-reported cardiorespiratory fitness: prediction and classification of risk of cardiovascular disease mortality and longevity--a prospective investigation in the Copenhagen City Heart Study. J Am Heart Association 2015;27:e001495. http://dx.doi.org/10.1161/ JAHA.114.001495.

12. Schnohr P, Jensen G, Lange H, Scharling H, Appleyard M. The Copenhagen City Heart Study. Tables with data from the third Examination 1991-1994. Europ Heart J 2001;Supplement:H1-H83

13. Schnohr P. Physical activity in leisure time: impact on mortality. Risks and benefits. Dan Med Bull 2009;56:40-71.

14. Lin DY, Wei LJ, Ying Z. Checking the Cox model with cumulative sums of martingale-based residuals. Biometrika 1993;80:557-572. http://dx.doi.org/10.1093/biomet/80.3.557

15. Holtermann A, Mortensen OS, Burr H, Søgaard K, Gyntelberg F, Suadicani P. The interplay between physical activity at work and during leisure time - risk of ischemic heart disease and allcause mortality in middle-aged Caucasian men. Scand J Work Environ Health 2009;35:466-474. http://dx.doi.org/10.5271/ sjweh. 1357

16. Holtermann A, Marott JL, Gyntelberg F, Søgaard K, Suadicani $\mathrm{P}$, Mortensen OS, et al. Occupational and leisure time physical activity: risk of all-cause mortality and myocardial infarction in the Copenhagen City Heart Study. A prospective cohort study. BMJ Open 2012;13(2):e000556. http://dx.doi. org/10.1136/bmjopen-2011-000556

17. Allesøe K, Holtermann A, Aadahl M, Thomsen JF, Hundrup YA, Søgaard K. High occupational physical activity and risk of ischaemic heart disease in women: The interplay with physical activity during leisure time. Europ J Prev Cardiol 2015;22(12):1601-1608. http://dx.doi. org $/ 10.1177 / 2047487314554866$
18. Harari G, Green M, Selber-Sagi S. Combined association of occupational and leisure-time physical activity with all cause and coronary heart disease mortality among a cohort of men followed-up for 22 years. Occup Environ Med 2015; 72(9):617624. http://dx.doi.org/10.1136/oemed-2014-102613

19. Blair SN, Kampert JB, Kohl HW, Barlow CE, Macera CA, Paffenbarger RS, et al. Influences of cardiorespiratory fitness and other precursors on cardiovascular disease and all-cause mortality in men and women. J Am Med Association 1996;276(3):205-210. http://dx.doi.org/10.1001/ jama.1996.03540030039029

20. Krause N, Brand RJ, Kaplan GA, Kauhanen J, Malla S, Tuomainen T-P, et al. Occupational physical activity, energy expenditure and 11-year progression of carotid atherosclerosis. Scand J Work Environ Health 2007;33(6):405-424. http:// dx.doi.org/10.5271/sjweh.1171

21. Clays E, De Bacquer D, Van Herck K, De Backer G, Kittel F, Holtermann A. Occupational and leisure time physical activity in contrasting relation to ambulatory blood pressure. BMC Publ Health 2012;12:1002. http://dx.doi.org/10.1186/14712458-12-1002

22. Ilmarinen J. Work and cardiovascular health: viewpoint of occupational physiology. Ann Med 1989;21(3):209-214. http://dx.doi.org/10.3109/07853898909149935

23. Holtermann A. Occupational and leisure-time physical activity and coronary heart disease. Occup Environ Med 2015;72(9):615-616. http://dx.doi.org/10.1136/ oemed-2015-102933

24. Woods V, Buckle P. Musculoskeletal ill health amongst cleaners and recommendations for work organisational change. Int J Ind Ergonom 2006;36(1):61-72. http://dx.doi. org/10.1016/j.ergon.2005.08.001

25. Gram B, Holtermann A, Søgaard K, Sjøgaard G. Effect of individualized worksite exercise training on aerobic capacity and muscle strength among construction workers - a randomized controlled intervention study. Scand J Work Environ Health 2012;38(5):467-475. http://dx.doi. org/10.5271/sjweh.3260

26. Korshøj M, Lidegaard M, Skotte J, Krustrup P, Krause N, Søgaard K, et al. Does aerobic exercise improve or impair cardiorespiratory fitness and health among cleaners? A cluster randomized controlled trial. Scand J Work Environ Health 2015;41(2):140-152. http://dx.doi.org/10.5271/sjweh.3475

27. Sallis JF, Saelens BE. Assessment of physical activity by selfreport: Status, limitations, and future directions. Res Quart Exerc And Sport 2000;71(2):S1-S14. http://dx.doi.org/10.10 80/02701367.2000.11082780

Received for publication: 6 August 2015 